\title{
PAPER \\ CU-MAC: A MAC Protocol for Centralized UAV Networks with Directional Antennas
}

\author{
Aijing $\mathrm{LI}^{\dagger, \dagger \dagger \mathrm{a})}$, Nonmember, Guodong $\mathrm{WU}^{\dagger \dagger}$, Member, Chao DONG ${ }^{\dagger \dagger \dagger}$, and Lei $\mathrm{ZHANG}^{\dagger \dagger}$, Nonmembers
}

\begin{abstract}
SUMMARY Media Access Control (MAC) is critical to guarantee different Quality of Service (QoS) requirements for Unmanned Aerial Vehicle (UAV) networks, such as high reliability for safety packets and high throughput for service packets. Meanwhile, due to their ability to provide lower delay and higher data rates, more UAVs are using frequently directional antennas. However, it is challenging to support different QoS in UAV networks with directional antennas, because of the high mobility of UAV which causes serious channel resource loss. In this paper, we propose CU-MAC which is a MAC protocol for Centralized UAV networks with directional antennas. First, we design a mobility prediction based time-frame optimization scheme to provide reliable broadcast service for safety packets. Then, a traffic prediction based channel allocation scheme is proposed to guarantee the priority of video packets which are the most common service packets nowadays. Simulation results show that compared with other representative protocols, CU-MAC achieves higher reliability for safety packets and improves the throughput of service packets, especially video packets.

key words: MAC, UAV networks, quality of service, directional antennas
\end{abstract}

\section{Introduction}

With the miniaturization and low-cost of Unmanned Aerial Vehicle (UAV), UAV networks have attracted much attention for their ability to support multi-UAVs cooperation effectively. In this paper, we focus on UAV networks where a centralized control UAV can communicate with all the other UAVs in the networks. This centralized network structure is widely applied now, for example, UAV sensor networks [1][3]. As shown in Fig. 1, a centralized sink UAV is receiving service information, e.g., video, image or other sensing data, from the other sensing UAVs. In addition, to guarantee flight safety, all the UAVs have to broadcast safety information to nearby UAVs. For safety packets broadcast, low delay and high reliability are required. But for service packets unicast, high throughput is more desired. Therefore, providing different Quality of Service (QoS) guarantees for safety and service packets is critical for the development of UAV net-

Manuscript received April 16, 2019.

Manuscript revised September 4, 2019.

Manuscript publicized November 6, 2019.

$\dagger$ The author is with the Department of Computer Science and Engineering, Shanghai Jiao Tong University, Shanghai 200240, China.

${ }^{\dagger \dagger}$ The authors are with the College of Communications Engineering, Army Engineering University of PLA, Nanjing 210007, China.

${ }^{+\dagger \dagger}$ The author is with the College of Electronic and Information Engineering, Nanjing University of Aeronautics and Astronautics, Nanjing, 210016, China.

a)E-mail: lishan_wh@126.com

DOI: 10.1587/transcom.2019EBP3089

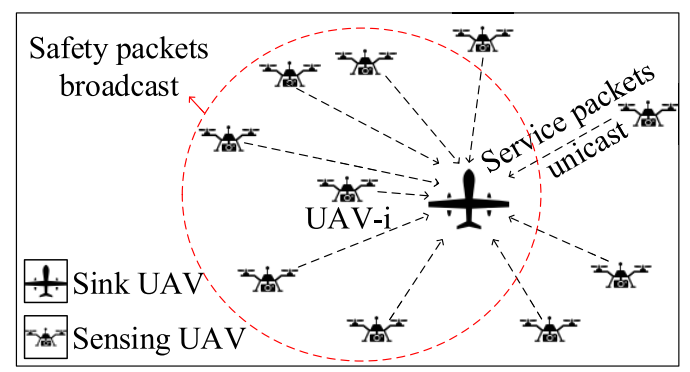

Fig. 1 UAV sensor networks.

works.

Just like traditional wireless networks, Medium Access Control (MAC) is essential to guarantee different QoS requirements for UAV networks. Meanwhile, to provide lower delay and higher data rate, directional antennas are being more widely installed on UAVs [1]. However, the use of directional antennas and the high mobility of UAVs bring some challenges for the MAC protocol of UAV networks. Firstly, UAVs are flying at high velocity, which causes frequent link interruption. The channel resource loss is more serious with the use of directional antennas because the beam angle is narrow, which makes the alignment difficult. Secondly, as a special type of service packets, video packets are frequently uploaded in many UAV scenarios and should be given higher priority than other service packets [4]. In conclusion, designing a MAC protocol to provide different QoS guarantees (especially higher priority for video packets) is an important and challenging goal for UAV networks with directional antennas.

Nowadays, most MAC protocols [5]-[7], [12] for UAV networks are contention-based, which will cause serious collisions with the expansion of the network scale. Meanwhile, most works [5]-[7], [12]-[14] think little about the impact of UAV mobility, which leads to frequent link interception and channel resource loss. Therefore, in UAV networks with directional antennas, how to provide different QoS guarantees under the high mobility of UAVs needs to be further studied. Fortunately, we observed that UAV trajectories are usually predictable. To maintain the stability of UAVs, UAV trajectories are always smooth [15], that is, the velocity and direction will not change rapidly in a short time. Taking the mobility model of UAVs into consideration, we pursue the optimization of the MAC protocol of UAV networks.

In this paper, we propose a MAC protocol called CU- 
MAC to provide different QoS guarantees for Centralized UAV networks with directional antennas. CU-MAC provides reliable broadcast service for safety packets based on dynamic Time Division Multiple Access (TDMA), and then allocates channel resources to UAVs according to the queue length of service packets to provide higher priority for video packets. Specific contributions can be summarized as follows:

(1) We propose CU-MAC which utilizes a mobility prediction based time-frame optimization scheme to overcome channel resource loss and provide reliable broadcast service for safety packets.

(2) Combining mobility prediction and traffic prediction, we propose a channel allocation scheme to guarantee the priority of video packets.

(3) Simulation results show that compared with other protocols, CU-MAC achieves higher Packet Delivery Rate (PDR) for safety packets, and maintains the delay in an acceptable range. Moreover, CU-MAC guarantees the priority of video packets while improves the throughput of service packets.

The rest of this paper is organized as follows. Related works are discussed in Sect. 2 . We describe the system model of CU-MAC in Sect. 3. Section 4 details the mechanism of CU-MAC. Performance evaluation is presented in Sect. 5. Section 6 concludes this paper.

\section{Related Works}

Because of the advantages of directional antennas, most of the MAC protocols designed for FANETs adopt directional antennas at present. The experience of MAC protocols in VANETs is also used in FANET. Enhance Distribution Coordinate Access (EDCA) mechanism is adopted in IEEE 802.11p [8]. EDCA offers control packets to avoid hiddenterminal problem. However, the handshake scheme cannot be implemented when vehicles broadcast safety packets. A centralized scheduling protocol was proposed for VANETs [9]. It uses Road Side Unit (RSU) to collect vehicle information and makes schedule decisions based on channel quality, speed, and access control. PT-MAC [10] is a TDMA protocol based on a novel way of predicting encounter collisions according to the information of road and vehicles within three hops. In [11], VAT-MAC was proposed to improve the performance in terms of scalability and throughput. But these works depend on RSU, which can not be applied in FANET.

Alsbatat et al. proposed an adaptive directional MAC protocol called AMUAV for UAV networks [5]. First, control packets are transmitted to exchange location information by omnidirectional antennas, and then other packets are exchanged with directional antennas. AMUAV is contention based and the antennas need to switch frequently. To solve the deafness problem of directional antennas in AMUAV, Abdel Ilah et al. presented LODMAC [6], which requires UAVs to be equipped with two independent transceivers. Although LODMAC can overcome the deafness problem, it also increases the hardware burden placed on UAVs. Zhigao Zheng et al. proposed PPMAC [7] which combines position estimation and directional antennas to avoid link interruption. But PPMAC ignores the narrow beam angle of directional antennas, and it also requires UAVs to be equipped with two independent transceivers. Duc Dang et al. proposed MMAC-DA [16], a multi-channel MAC protocol based on directional antenna, which is the first MAC protocol that can support both directional antenna and multichannel. The CSMA/CA mechanism adopted by MMACDA on the control channel cannot provide reliable guarantee for the security packets. Besides, the mobility of the node not only poses a challenge to the alignment of the directional antenna, but also causes a link interruption problem in the transmission process. In generally, such MAC protocols [5]-[7] for UAV networks are contention-based and think little about the link interception issue caused by the mobility of UAVs. As a result, the channel resource loss caused from channel contention and link interception will cut down the performance of UAV networks.

In addition, a new token-based MAC protocol [13] with full duplex and multi-packet reception radio was proposed, which requires UAVs to update Channel State Information (CSI) frequently. The performance results showed that the proposed MAC protocol is efficient if the CSI is perfect. However, the frequent updating of CSI increase the network overhead. In addition, it assumes that UAVs have the ability of full duplex communication and multi-packet reception, which makes its application scenarios limited. A CollisionFree MAC Protocol named CF-MAC [14] was proposed for UAV Ad hoc networks, which utilizes a region marking scheme to reduce the packet collisions probability, but it ignores the packet collisions caused by the mobility of UAVs. Our former work FM-MAC [21] proposed a multi-channel MAC protocol for distributed FANETs, which can provide better performance for video traffic by a preemptive mechanism. However, it works in distributed networks and causes more overhead for channel coordination. Besides, there may be collisions when channel coordination fails.

Thus, the research of MAC protocol for UAV networks is not mature, and several problems are still need to be further studied. First, the high mobility feature of UAVs is not taken into full consideration, which can greatly reduce channel access performance. Second, current research ignores the differences of QoS requirements from different applications. So it is necessary to conduct research on the MAC protocol design for UAV networks.

\section{System Model}

\subsection{Network Model}

In this paper, as shown in Fig. 1, there is a sink UAV, i.e., centralized UAV, to communicate with all the other UAVs. To guarantee flight safety, each UAV needs to broadcast safety packets periodically, e.g., UAV-i in Fig. 1. We assume that all UAVs are equipped with identical antenna which can 


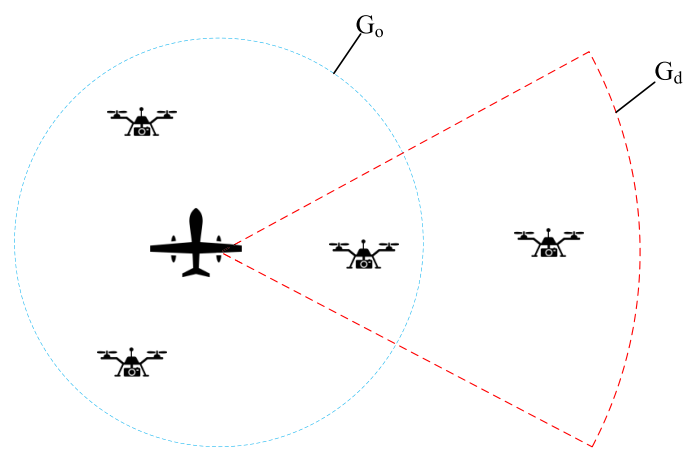

Fig. 2 Omnidirectional mode and directional mode model.

\begin{tabular}{|c|c|c|c|}
\hline MAC Header & \multicolumn{3}{|c|}{ Packet Payload } \\
\hline Position & Velocity & Queue length & Type \\
\hline 10 bytes 1 byte & 1 byte 1 bit
\end{tabular}

Fig. 3 MAC frame structure of safety packets.

be synchronized to a common time system. As in [16], the antennas can operate with either omnidirectional mode or directional mode. As is shown in Fig. 2, antenna working in omnidirectional mode radiates equally in all directions to provide the widest possible signal coverage. A directional antenna is designed to radiate sufficiently in a particular direction, resulting in a limited coverage area. The antenna gain in omnidirectional and directional modes are $G_{o}$ and $G_{d}$, respectively. In CU-MAC, omnidirectional mode is used to broadcast safety packets, while the directional mode is for service packets unicast.

\subsection{Frame Structure}

Location information is a necessary condition for directional antenna communication. Considering that UAVs also need to regularly broadcast location information for flight safety, as shown in Fig. 3, CU-MAC adds several fields to the MAC frame structure of safety packets, including position, velocity, the queue length and type of service packets. Position and velocity are used for directional antennas aligning and mobility prediction of UAVs. The queue length and type of service packets are used to formulate the channel allocation scheme. If there are no cached service packets in the queue, the queue length and type of service packets are set to 0 . In this way, each UAV can get the neighbors' position and velocity, which brings only about 12 bytes extra overhead for the MAC layer.

\section{CU-MAC}

In this section, we elaborate the design of CU-MAC. First, combing with the time-frame structure, we overview the operation of CU-MAC. Then, mobility prediction based time-frame optimization scheme and traffic prediction based channel allocation scheme are presented.

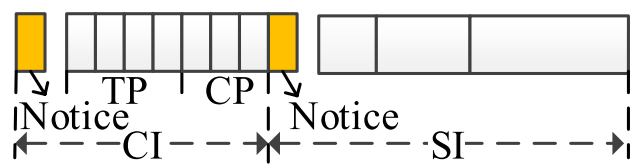

Fig. 4 Time-frame structure of CU-MAC.

\subsection{Overview}

As shown in Fig. 4, different from other works [5]-[7], [12], CU-MAC further divides the time-frame into Control Interval (CI) and Service Interval (SI). During CI, the antenna should be set to omnidirectional mode, and all UAVs broadcast safety packets based on TDMA. Specifically, CI is further divided into Notice, Transmission Phase (TP) and Contention Phase (CP). At the beginning of CI, centralized UAV broadcasts a notice message which includes the following information: (1) time slots allocation plan in TP which is used for the UAVs that have already accessed the networks; (2) the number of contending time slots in $\mathrm{CP}$ which is used for the UAVs that have attempts to access the networks; (3) its own safety packets. Based on mobility prediction, the length of CI can be dynamically adjusted to improve the channel utilization. Subsequently, each UAV broadcasts their safety packets in the corresponding slots according to the notice message of centralized UAV. At the beginning of SI, centralized UAV broadcasts a notice message again to allocate the channel resources (i.e. transmission time) of SI according to the known queue length of service packets on UAVs. Then UAVs switch their antennas to directional mode, adjust the beam direction according to the position information in the safety message, and send service packets according to the allocation result. To control the delay of safety packets, each time-frame is fixed to $100 \mathrm{~ms}$. UAVs can get the time frame synchronization according to the local clock and Pulse Per Second (PPS) from GPS.

\subsection{Mobility Prediction Based Time-Frame Optimization}

In this paper, we assume the moving mode of UAV is a smooth moving model, which means the speed and direction of UAVs will not change greatly in a short time. To overcome the high mobility of UAVs and improve the channel utilization, CU-MAC optimizes the length of CI dynamically. Based on the predicted number of leaving and new arriving UAVs, CU-MAC adjusts the number of slots in CI for the next time-frame. We assume that the current time frame is the $n$-th time-frame, and we need to calculate the slots in CI at the $(n+1)$-th time-frame. First of all, we should calculate the number of UAVs in the networks:

$$
N(n)=S_{t p}(n)+N_{n}(n-1),
$$

where $N(n)$ is the number of UAVs at the $n$-th time-frame, and $S_{t p}(n)$ is the number of slots in TP. $N_{n}(n-1)$ denotes the number of newly arrived UAVs at the $(n-1)$-th frame which attempt to access the channel at the $n$-th time-frame, because 
new nodes will not know the slots of $\mathrm{CP}$ until they receive the notice message. Assuming that the access of new UAVS to the idle time slot satisfies a uniform distribution, $N_{n}(n-$ 1) can be obtained according to the ratio of the number of collision slots to the number of total slots in CP [17]:

$$
\frac{S_{c p-c}(n)}{S_{c p}(n)}=1-\left(1-\frac{1}{S_{c p}(n)}\right)^{N_{n}(n-1)}\left(1+\frac{N_{n}(n-1)}{S_{c p}(n)-1}\right),
$$

where $S_{c p-c}(n)$ and $S_{c p}(n)$ represent the number of collision slots and the number of total slots in $\mathrm{CP}$ at the $n$-th time-frame, respectively. As $S_{c p}(n)$ and $S_{c p-c}(n)$ are known values by centralized UAV, $N_{n}(n-1)$ and $N(n)$ can be derived by equation (2) and (1) successively.

Then, CU-MAC estimates the number of newly arriving UAVs at the $n$-th time-frame, i.e., $\hat{N}_{n}(n)$, which can be estimated with the mean of past data:

$$
\hat{N}_{n}(n)=\frac{1}{m} \sum_{i=1}^{m} N_{n}(n-i),
$$

where $m$ represents the number of past data.

Finally, CU-MAC uses mobility prediction to calculate the number of UAVs that leave the communication range (under omnidirectional mode) of centralized UAV at the $(n+$ $1)$-th time-frame, i.e., $N_{\text {leave }}(n)$. As the trajectory of UAVs is smooth, $N_{\text {leave }}(n)$ is calculated as follows:

$$
\begin{gathered}
N_{\text {leave }}=\sum\left(1-x_{i}\right) \\
\text { s.t. } x_{i}=\left\{\begin{array}{l}
0, d\left(P_{c}+\vec{V}_{c} t_{S I}, P_{i}+\vec{V}_{i} t_{S I}\right)>R_{o} \\
1, \text { otherwise }
\end{array},\right.
\end{gathered}
$$

where $P_{c}$ and $P_{i}$ are the current position of centralized UAV and UAV-i, respectively, which are expressed by their 3dimensional coordinates. $d\left(P_{c}, P_{i}\right)$ indicates the distance between centralized UAV and UAV-i. $\vec{V}_{c}$ and $\vec{V}_{i}$ indicate the current velocity of centralized UAV and UAV-i, and $t_{S I}$ represents the current length of SI. The range of centralized networks is depending on the communication distance of omnidirectional antenna which is expressed as $R_{o}$. If $x_{i}$ equals $0, \mathrm{UAV}-\mathrm{i}$ will leave the range of centralized networks at the next time-frame.

Because of the rapid velocity of UAVs, it is frequent that some UAVs leave or connect to the centralized networks. At the next time-frame, it is unnecessary to allocate slots for those UAVs which will leave the networks. In addition, CU-MAC adjusts the number of slots in CP at the $(n+1)$-th time-frame, which enables new UAVs to access the networks. Therefore, the number of slots at the next CI can be calculated as follows:

$$
\begin{aligned}
S_{C I}(n+1) & =S_{t p}(n+1)+S_{c p}(n+1) \\
& =N(n)-N_{\text {leave }}(n)+N_{n}(n),
\end{aligned}
$$

where $S_{C I}(n+1)$ is the number of slots at the $(n+1)$-th timeframe. $S_{t p}(n+1)$ is the number of TP slots at the $(n+1)$ th time-frame, which will be assigned to UAVs that have already accessed the channel at the $n$-th time-frame. Thus, $S_{C I}(n+1)$ can be calculated by

$$
S_{t p}(n+1)=S_{t p}(n)+S_{c p-s}(n)-N_{\text {leave }}(n),
$$

where $S_{c p-s}(n)$ represents the number of slots that have been successfully accessed by new UAVs at the $n$-th time-frame. $N_{\text {leave }}$ represents the number of UAVs that access channel in $\mathrm{TP}$ at the $n$-th time-frame but will leave the network at the $(n+1)$-th frame.

$$
S_{c p}(n+1)=N_{n}(n-1)-S_{c p-s}(n)+N_{n}(n),
$$

$S_{c p}(n+1)$ is the number of CP slots at the $(n+1)$-th timeframe, which are used for contention for new UAVs. It is the total number of UAVs that fail to access the channel at the $n$-th frame $\left(N_{n}(n-1)-S_{c p-s}(n)\right)$ and the number of newly arrived UAVs at the n-th frame $\left(N_{n}(n)\right)$. Taking Eqs. (1) (3) (4) into Eqs. (5) (6) (7), centralized UAV can calculate the number of slots at the next CI. Note that mobility prediction is not completely reliable, there may be a small probability event that a UAV is predicted to leave the network, but actually not. In this case, the UAV needs to compete for free time slots in the CP of next time-frame.

\subsection{Traffic Prediction Based Channel Allocation Scheme}

After CI, centralized UAV obtains the queue-length of service packets of all UAVs, and allocates channel resources for them. To guarantee enough bandwidth for video packets and prevent link interruption, CU-MAC develops a channel allocation scheme based on mobility prediction and traffic prediction. Centralized UAV first determines the UAVs which will trigger link interruption, and cancels the allocation of channel resources for these UAVs. According to the Law of Cosines, the formula is as follows:

$$
\begin{aligned}
& x_{i}=0, \text { if }\left\{\begin{array}{l}
\cos ^{-1} \frac{\overrightarrow{P_{c} P_{i}} \cdot \overrightarrow{\left(P_{c}+\vec{V}_{c} t_{S I}\right)\left(P_{i}+\vec{V}_{i} t_{S I}\right)}}{\left|\overrightarrow{P_{c} P_{i}}\right| \mid\left(\overrightarrow{\left.P_{c}+\vec{V}_{c} t_{S I}\right)\left(P_{i}+\vec{V}_{i} t_{S I}\right)} \mid\right.} \geq \frac{\theta}{2} \text {, and } \\
d\left(P_{c}+\vec{V}_{c} t_{S I}, P_{i}+\vec{V}_{i} t_{S I}\right)>R_{d}
\end{array}\right. \\
& x_{i}=1 \text {, otherwise, }
\end{aligned}
$$

where $R_{d}$ and $\theta$ indicate the communication distance and angle of directional antennas. In contrast to formula (4), formula (8) modifies distance constraint and adds angle constraint under directional mode. If $x_{i}$ equals $0, \mathrm{UAV}-\mathrm{i}$ can not maintain communications with centralized UAV in the coming SI, even though the antennas are in directional mode.

Then, CU-MAC predicts the arrival traffic of service packets at the next time-frame to complete the channel allocation scheme. Assuming that there are $N$ UAVs in the networks, $R_{i}(n)$ indicates the queue-length of service packets of UAV-i at the $n$-th time-frame $\left(R_{i} \geq 0\right)$, which is composed of packets with fixed size. The queue-length of UAV-i in previous $m$ time-frames are presented as $R_{i}(n-1), R_{i}(n-2)$, $\ldots, R_{i}(n-m)$. The data arrival rate of UAVs at each timeframe can be calculated. For example, we can get the arrival 
rate of UAV-i at the $n$-th time-frame (expressed as $\left.r_{i}(n)\right)$ as follows:

$$
r_{i}(n)=R_{i}(n)-\left[R_{i}(n-1)-T_{i}(n-1)\right],
$$

where $T_{i}(n-1)$ represents the packet size that UAV-i has successfully transmitted at the $(n-1)$-th time-frame. Similarly, CU-MAC can get the packet arrival rate of each timeframe $\left(r_{i}(n), r_{i}(n-1), r_{i}(n-2), \ldots, r_{i}(n-m+1)\right)$. We assume that the arrival rate of service packets is correlated in time. According to the arrival rate of the previous frames, i.e., $\left\{r_{i}(n), r_{i}(n-1), r_{i}(n-2), \ldots, r_{i}(n-m+1)\right\}$, the arrival rate $r_{i}(n+1)$ can be predicted by traffic prediction theory, which models the relationship between the traffic volume and simple statistics about flows using a Hidden Markov Model [18]. Instead of direct measurement of the traffic volume, we estimate and predict the hidden traffic volume based on those simple flow statistics.

When the channel resources of SI can not satisfy all UAVs' requirements, the bandwidth of video packets should be guaranteed firstly. Assuming that there are $k$ UAVs to transmit video packets at the $n$-th time-frame. We define the packet size of UAVs as $R_{v 1}(n), R_{v 2}(n), R_{v 3}(n), \ldots, R_{v k}(n)$, respectively. According to the minimum bandwidth requirements $B_{\text {min }}$ (the minimum transmission rate that guarantees video fluency), the shortest transmission time for each UAV (the minimum length of time needed to allocate in each time-frame, which are expressed as $t_{v 1 \text { min }}, t_{v 2 \text { min }}, t_{v 3 \text { min }}, \ldots$, $\left.t_{v k m i n}\right)$ can be calculated as follows:

$$
t_{v j \min }(n)=\frac{x_{j} \frac{R_{v j}(n)}{B_{t}}}{\max \left(1,\left\lfloor\frac{R_{v j}(n)}{B_{\min } T}\right\rfloor\right)},
$$

where $T$ represents the total time of one time-frame and $B_{t}$ represents channel capacity. The calculated transmission time is an initial value and needs to be adjusted according to the channel conditions at the next time-frame. If the current channel is more congested than the channel of next time-frame, the transmission time allocated for video packets should be reduced, which makes more channel resources available for other types of traffic. If the channel of next time-frame is more congested than that of current timeframe, more transmission time should be allocated for video packets. Therefore, the transmission time allocated to the UAVs which need to send video packets is:

$$
\begin{array}{r}
t_{v j}(n)=t_{v j \min }(n) \alpha \frac{t_{S I}(n) \sum_{i=1}^{N} x_{i} r_{i}(n+1)}{t_{S I}(n+1) \sum_{i=1}^{N} x_{i} r_{i}(n)} \\
\text { s.t. }\left\{\begin{array}{l}
t_{S I}(n)<\sum_{i=1}^{N} \frac{x_{i} R_{i}(n)}{B_{t}} \\
j=1,2 \ldots, k
\end{array}\right.
\end{array}
$$

where $\alpha$ is correction factor, which is a coefficient of the algorithm. We use two factors to measure the congestion of SI at the next time-frame. The first one is the ratio between the data arrival rate of the next time-frame and the current timeframe. And the other one is the ratio of SI length at current and the next time-frame. As is known in Sect. 4.2, the length of CI can adjust dynamically. Thus, the length of SI at the next time-frame will be modified accordingly. Meanwhile, CU-MAC also combines the mobility prediction of UAVs. If UAV-i can not maintain communications with centralized UAV in $S I(n), x_{i}$ equals 0 . Otherwise $x_{i}$ equals 1 .

In an extreme condition, the current channel resources can not meet the minimum requirements of all video packets, i.e., $\sum_{i=1}^{k} t_{\text {vimin }}(n)>t_{S I}$. The greedy algorithm is used to ignore one or more UAVs that have the least impact, which is shown in Algorithm 1. After ensuring the bandwidth of video packets, CU-MAC assigns the transmission time for other service packets, which is proportional to their queue length if there is no enough channel resources. The formula is as follows:

$$
\begin{gathered}
t_{i}=\left(t_{S I}-\sum t_{v j}(n)\right) \frac{R_{i}(n) x_{i}}{\sum R_{j}(n) x_{j}} \\
\text { s.t. }\left\{\begin{array}{l}
v j \in C \\
i, j \in(S-C),
\end{array}\right.
\end{gathered}
$$

where $t_{i}$ represents the channel resources allocated to the $i$ th non-video service. The set $C$ represents the UAVs that transmit video packets, and $S$ represents the set of all UAVs.

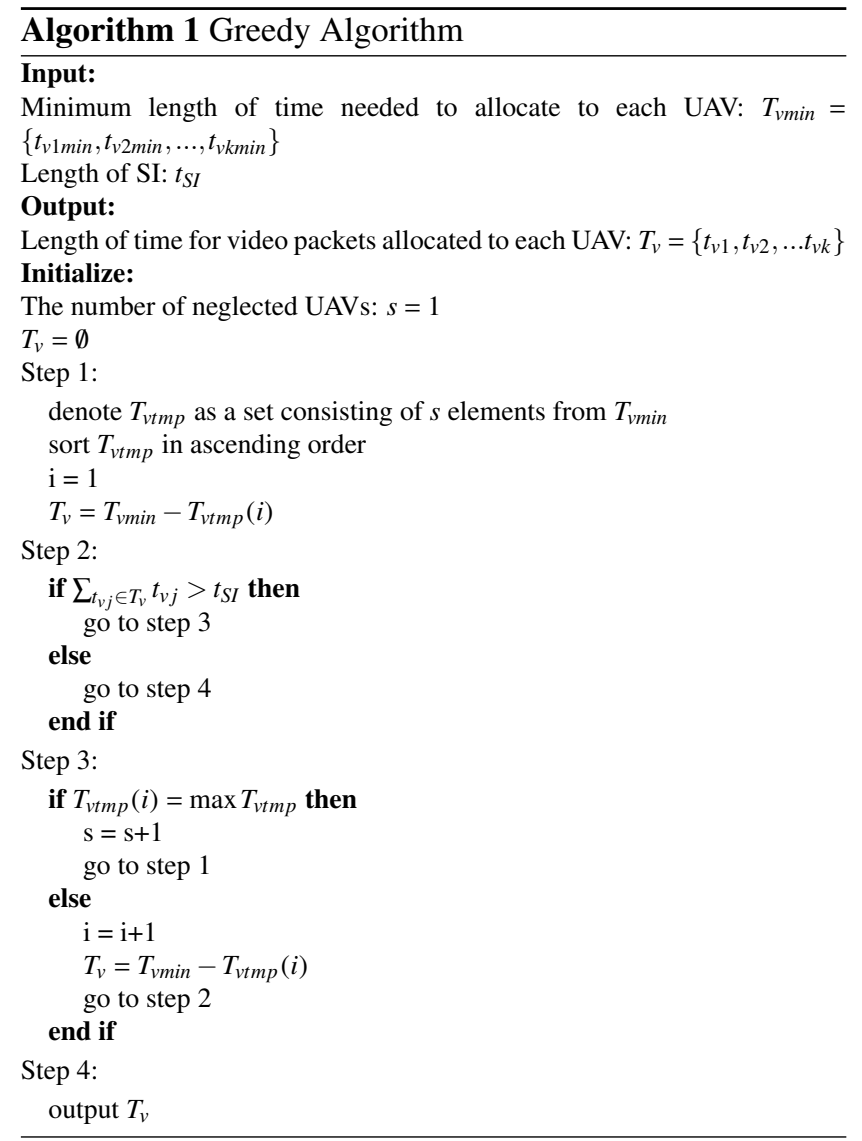

\section{Performance Evaluation}

We use OPNET to evaluate the performance of CU-MAC 
Table 1 Simulation parameters.

\begin{tabular}{|c|c|c|c|}
\hline \hline Parameter & Value & Parameter & Value \\
\hline Simulation scene & $5^{*} 5 * 2 \mathrm{~km}^{3}$ & $\begin{array}{c}\text { communication } \\
\text { distance of } R_{o} \text { and } R_{d}\end{array}$ & $0.5 / 1 \mathrm{~km}$ \\
\hline Flying velocity & $20-100 \mathrm{~m} / \mathrm{s}$ & Time-frame & $100 \mathrm{~ms}$ \\
\hline Date rate & $6 \mathrm{Mbps}$ & Safety packet size & 256 bytes \\
\hline Slot time in CI & $0.5 \mathrm{~ms}$ & Service packet size & $1.5 \mathrm{k}$ bytes \\
\hline$\theta$ & $60^{\circ}$ & Video packet size & $1-2 \mathrm{k}$ bytes \\
\hline Mobility model & Smooth & $m$ & 20 \\
\hline Video packets rate & 5 packets/s & Service packets rate & 7 \\
& & & \\
packets/s
\end{tabular}

and compare it with two representative protocols: AMUAV [5] and PPMAC [7]. The main simulation settings are summarized in Table 1. The coverage distance of the antenna in omnidirectional mode and directional mode is $0.5 \mathrm{~km}$ and $1 \mathrm{~km}$ respectively. The coverage angle of directional mode is set to $60^{\circ}$. We set each UAV with a random smooth mobility model [19]. Service and video traffic classes are generated by self-similar models [22] to better model real traffic. Different types of packets focus on different performance indicators. We evaluate the delay and PDR performance for safety packets, and the throughput performance for service packets.

Figure 5 and Fig. 6 show the delay and PDR of safety packets versus the number of UAVs respectively. The average velocity of UAVs is set to $20 \mathrm{~m} / \mathrm{s}$. As the number of UAVs increases, the delay of AMUAV and PPMAC rises sharply, while the delay of CU-MAC always stays at an acceptable range (about $0.05 \mathrm{~s}$ ) which satisfies the requirements of safety packets [20]. More importantly, CU-MAC always gets higher PDR (close to 1) than other protocols as shown in Fig. 6. For PPMAC and AMUAV, the collisions caused by competition are more frequent when the number of UAVs increases. However, in CU-MAC, contention-free $\mathrm{CI}$ avoids packet collisions, and time-frame optimization scheme can allocate reasonable slots for UAVs. The PDR of safety packets versus average velocity are displayed in Fig. 7. The number of UAVs is set to 20. As the average velocity of UAVs increases, the PDR of PPMAC and AMUAV decrease rapidly due to link interruption. However, the PDR of CU-MAC declines slowly, and is always higher than other protocols. Mobility prediction based time-frame avoids invalid transmission and keeps most slots available for effective UAVs.

From Fig. 8, we can find that even if PPMAC uses two transceivers (transmitting simultaneously), CU-MAC can achieve comparative throughput as PPMAC, and more than twice of AMUAV. When the average velocity of UAVs changes from $30 \mathrm{~m} / \mathrm{s}$ to $60 \mathrm{~m} / \mathrm{s}$, the throughput of PPMAC and AMUAV is reduced by $20 \%$, while the throughput of CU-MAC is only slightly affected. When the number and the velocity of UAVs increase, channel resources loss are more serious due to the mobility of UAVs in AMUAV and PPMAC. However, for CU-MAC, the mobility prediction based time optimization scheme and channel allocation scheme bring more available resources for SI. Figure 9

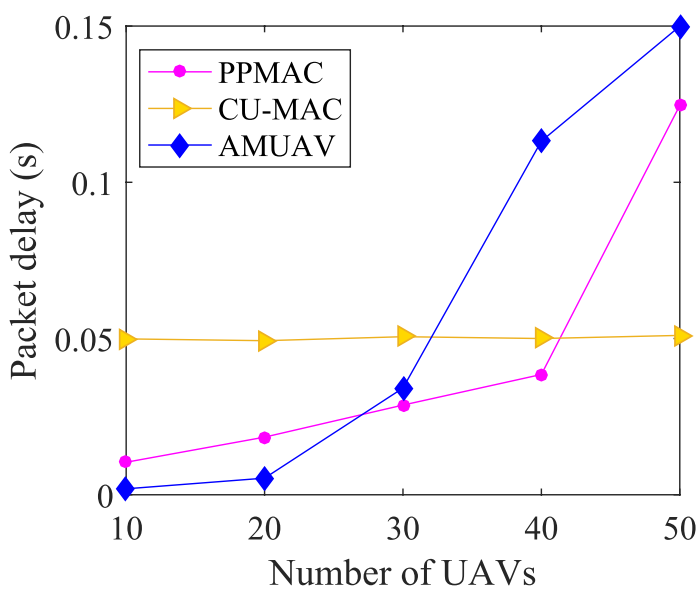

Fig. 5 Packet delay of safety packets versus the number of UAVs.

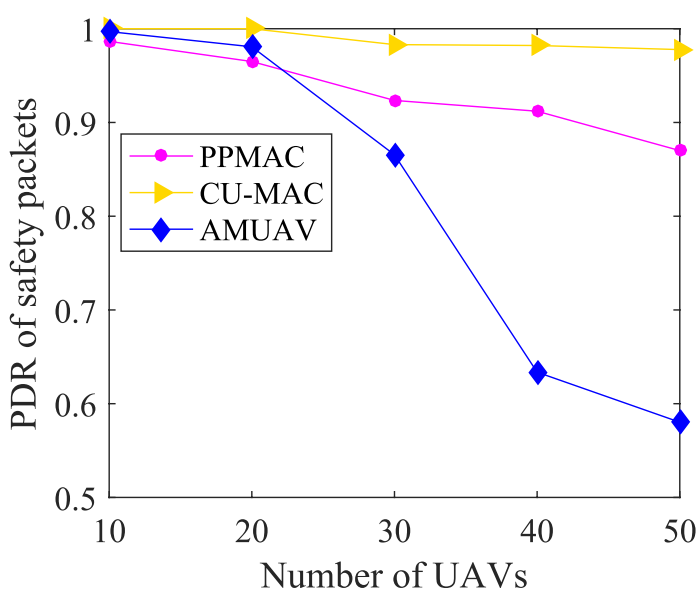

Fig. 6 PDR of safety packets versus the number of UAVs.

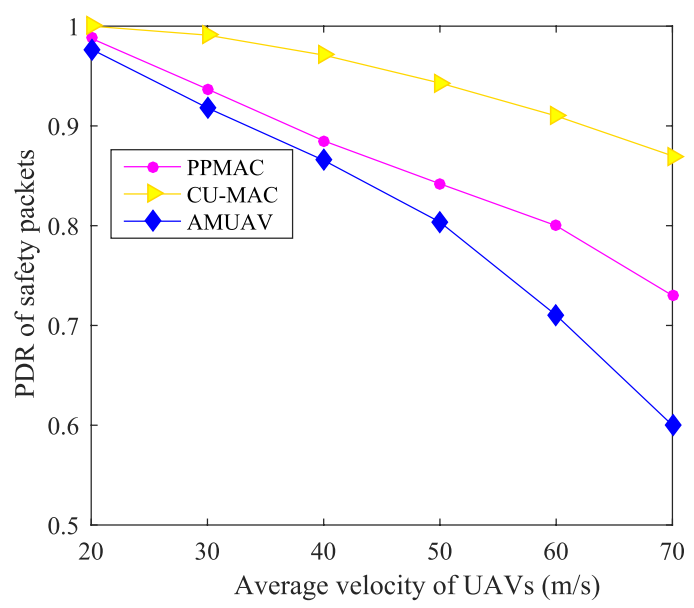

Fig. 7 PDR of safety packets versus average velocity of UAVs.

and Fig. 10 show the throughput of video packets versus the number of UAVs (packet size $=1500$ bytes) and packet size (number of UAVs $=30$ ). With the number of UAVs and packet size increases, the throughput of CU-MAC is almost linearly increasing and higher than other protocols. This is due to the traffic prediction based channel alloca- 


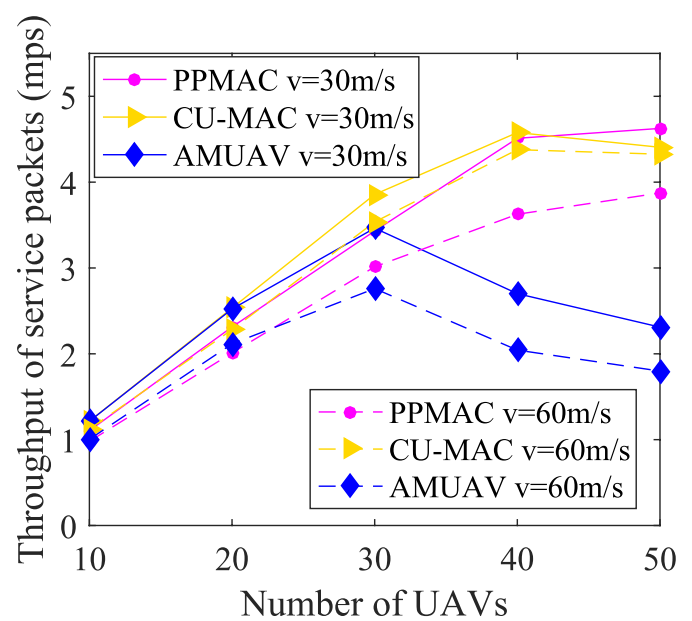

Fig. 8 Throughput of service packets versus the number of UAVs.

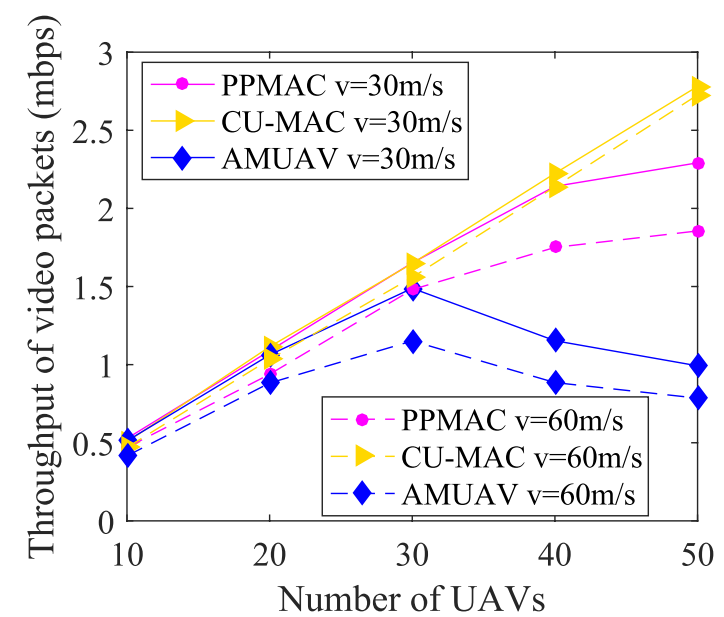

Fig. 9 Throughput of video packets versus the number of UAVs.

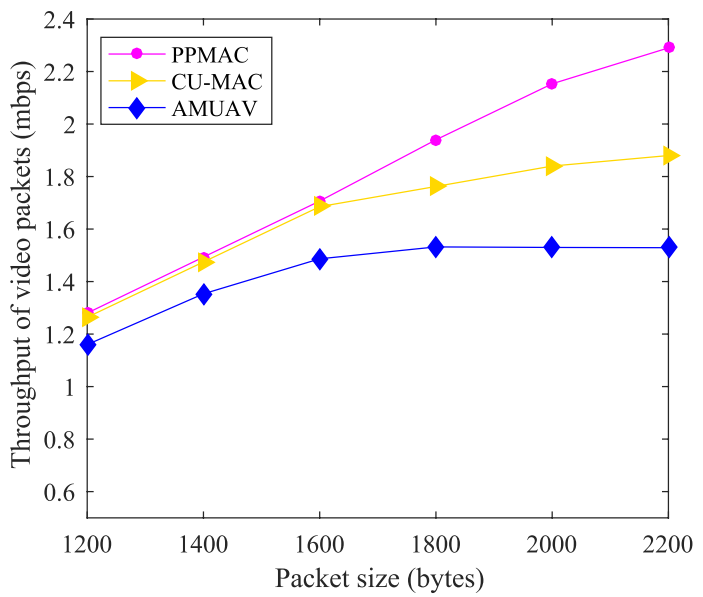

Fig. 10 Throughput of video packets versus packet size.

tion scheme, which can guarantee the transmission rate of video packets, especially when the channel resources are scarce. Through Fig. 10, we can observe although the average velocity changes from $30 \mathrm{~m} / \mathrm{s}$ to $60 \mathrm{~m} / \mathrm{s}$, the through- put of video packets of CU-MAC is only slightly affected. This is because channel allocation scheme also utilizes mobility prediction to prevent link interruption. As a result, it outperforms PPMAC and AMUAV in dynamic scenarios.

\section{Conclusion}

In this paper, we propose CU-MAC to provide different QoS guarantees for centralized UAV networks with directional antennas. Based on the proposed mobility prediction and traffic prediction schemes, CU-MAC dynamically optimizes the time-frame for safety packets and channel allocation for service packets. Simulations show that CU-MAC can not only ensure the reliability for safety packets, but also guarantee the priority for video packets while maintaining high throughput for service packets.

\section{Acknowledgments}

This work was supported in part by the National NSF of China under Grant 61472445, Grant 61631020, Grant 61702525 , and Grant 61702545 , and in part by the NSF of Jiangsu Province under Grant BK20140076.5.

\section{References}

[1] İ. Bekmezci, O. Sahingoz, and S. Temel, "Flying ad-Hoc networks (FANETs): A survey," Ad Hoc Networks, vol.11, pp.1254-1270, 2013. DOI: 10.1016/j.adhoc.2012.12.004

[2] T. Wu, P. Yang, Y. Yan, P. Li, and X. Rao, "Near optimal route association with Shannon model in multi-drone WSNs,' IEEE Access, vol.6, pp.60869-60880, 2018. DOI: 10.1109/ACCESS.2018.2874661

[3] T. Wu, P. Yang, H. Dai, P. Li, and X. Rao, "Near optimal bounded route association for drone-enabled rechargeable WSNs," Computer Networks, vol.145, no.9, pp.107-117, 2018. DOI: 10.1016/j.comnet.2018.07.004

[4] D. Rosario, J. Filho, D. Rosario, A. Santosy, and M. Gerla, "A relay placement mechanism based on UAV mobility for satisfactory video transmissions," Proc. Mediterranean Ad Hoc Networking Workshop, 2017. DOI: 10.1109/MedHocNet.2017.8001638

[5] A. Alshbatat and L. Dong, "Adaptive MAC protocol for UAV communication networks using directional antennas," Proc. International Conference on Networking, Sensing and Control (ICNSC), pp.598603, Chicago, 2010. DOI: 10.1109/ICNSC.2010.5461589

[6] S. Temel and I. Bekmezci, "LODMAC: Location oriented directional MAC protocol for FANETs," Computer Networks, vol.83, pp.76-84, 2015. DOI: 10.1016/j.comnet.2015.03.001

[7] Z. Zheng, A.K. Sangaiah, and T. Wang, "Adaptive communication protocols in flying ad hoc network," IEEE Commun. Mag., vol.56, no.1, pp.136-142, Jan. 2018. DOI: 10.1109/MCOM.2017.1700323

[8] D. Jiang and L. Delgrossi, "IEEE 802.11p: Towards an international standard for wireless access in vehicular environments," Proc. Vehicular Technology Conference (VTC), pp.2036-2040, Singapore, 2008. DOI: 10.1109/VETECS.2008.458

[9] R. Zhang, X. Cheng, L. Yang, X. Shen, and B. Jiao, "A novel centralized TDMA-based scheduling protocol for vehicular networks," IEEE Trans. Intell. Transp. Syst., vol.16, no.1, pp.411-416, 2015. DOI: $10.1109 /$ TITS.2014.2335746

[10] X. Jiang and D.H.C. Du, "PTMAC: A prediction-based TDMA MAC protocol for reducing packet collisions in VANET," IEEE Trans. Veh. Technol., vol.65, no.11, pp.9209-9223, 2016. DOI: 10.1109/TVT.2016.2519442 
[11] S. Cao and V.C.S. Lee, "A novel adaptive TDMA-based MAC protocol for VANETs," IEEE Commun. Lett., vol.22, no.3, pp.614-617, 2018. DOI: 10.1109/LCOMM.2017.2785378

[12] S. Say, H. Inata, and S. Shimamoto, "A hybrid collision coordination-based multiple access scheme for super dense aerial sensor networks," Proc. IEEE Wireless Communications and Networking Conference, pp.1-6, Doha, 2016. DOI: 10.1109/WCNC.2016.7565148

[13] Y. Cai, F.R. Yu, J. Li, Y. Zhou, and L. Lamont, "Medium access control for unmanned aerial vehicle (UAV) ad-hoc networks with full-duplex radios and multipacket reception capability," IEEE Trans. Veh. Technol., vol.62, no.1, pp.390-394, Jan. 2013. DOI: 10.1109/TVT.2012.2211905

[14] A. Jiang, Z. Mi, C. Dong, and H. Wang, "CF-MAC: A collisionfree MAC protocol for UAVs ad-hoc networks," Proc. IEEE Wireless Communications and Networking Conference, pp.1-6, 2016. DOI: 10.1109/WCNC.2016.7564844

[15] Y. Wan, K. Namuduri, Y. Zhou, and S. Fu, "A smooth-turn mobility model for airborne networks," IEEE Trans. Veh. Technol., vol.62, no.7, pp.3359-3370, Sept. 2013. DOI: 10.1109/TVT.2013.2251686

[16] D. Dang, H. Le, H.S. Kang, C. Hong, and J. Choe, "Multichannel MAC protocol with directional antennas in wireless ad hoc networks," Proc. International Conference on Information Networking (ICOIN), pp.81-86, Cambodia, 2015. DOI: 10.1109/ICOIN.2015.7057861

[17] Y. Park and H. Kim, "Application-level frequency control of periodic safety messages in the IEEE WAVE," Trans. Veh. Technol., vol.61, no.4, pp.1854-1862, 2012. DOI: 10.1109/TVT.2012.2190119

[18] Z. Chen, J. Wen, and Y. Geng, "Predicting future traffic using hidden Markov models," Proc. IEEE 24th International Conference on Network Protocols (ICNP), pp.1-6, Singapore, 2016. DOI: 10.1109/ICNP.2016.7785328

[19] A. Bujari, E. Claudio, and R. Daniele, "FANET application scenarios and mobility models," Proc. Workshop on Micro Aerial Vehicle Networks, pp.43-46, 2017. DOI: 10.1145/3086439.3086440

[20] "Enhanced LTE support for aerial vehicles," http://www.3gpp.org/ DynaReport/36-series.htm, accessed Dec. 14, 2017.

[21] G. Wu, C. Dong, A. Li, L. Zhang, and Q. Wu, "FM-MAC: A multichannel MAC protocol for FANETs with directional antenna," 2018 IEEE Global Communications Conference (GLOBECOM), pp.1-7, Abu Dhabi, United Arab Emirates, 2018.

[22] H.-D.J. Jeong, K. Pawlikowski, and D.C. McNickle. "Generation of self-similar processes for simulation studies of telecommunication networks," Math. Comput. Model., vol.38, no.11-13, pp.1249-1257, 2003.



Guodong Wu received his B.S. degree in communications engineering from Huazhong University of Science and Technology (HUST), Wuhan, China in 2015. He is currently pursuing a master degree at Army Engineering University of PLA. His current research interests include Vehicular Ad Hoc NETworks (VANETs) and Flying Ad Hoc NETworks (FANETs).

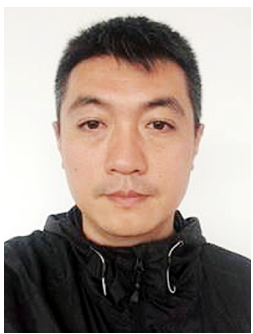

Chao Dong received his Ph.D. degree in Communication Engineering from PLA University of Science and Technology, China, in 2007. From 2008 to 2011, he worked as a postdoctoral at the Department of Computer Science and Technology, Nanjing University, China. $\mathrm{He}$ is now a full professor at the College of Electronic and Information Engineering, Nanjing University of Aeronautics and Astronautics. His current research interests include device-todevice communications, Unmanned Air Vehicles (UAVs) swarm networking and anti-jamming network protocol.

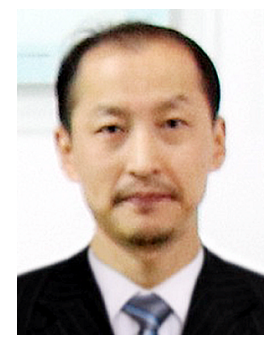

Lei Zhang received his Ph.D. degree in Communication Engineering from PLA University of Science and Technology in 2000. From 2001 to 2003, he conducted his postdoctoral research at the State Key Laboratory of Mobile Communications, Southeast University. He is now a full professor at the College of Communication Engineering, Army Engineering University of PLA. His major research interests include embedded operating system, fog computing, and ad hoc networks.



Aijing Li received her Ph.D. degree in Computer Science and Technology from PLA University of Science and Technology, Nanjing, China. She joined the College of Communication Engineering, Army Engineering University of PLA in 2015. Her current research interests include the areas of wireless communications, cognitive radio networking, and heterogeneous networking. 